\title{
Compact Lie Group Actions on Closed Manifolds of Non-positive Curvature
}

\author{
BIN XU \\ Department of Mathematics, Johns Hopkins UniVersity, BALtimore MD 21218, USA \\ E-mail: bxu@math.jhu.edu
}

\begin{abstract}
A. Borel proved that, if a finite group $F$ acts effectively and continuously on a closed aspherical manifold $M$ with centerless fundamental group $\pi_{1}(M)$, then a natural homomorphism $\psi$ from $F$ to the outer automorphism group Out $\pi_{1}(M)$ of $\pi_{1}(M)$, called the associated abstract kernel, is a monomorphism. In this paper, we investigate to what extent Borel's theorem holds for a compact Lie group $G$ acting effectively and smoothly on a particular orientable aspherical manifold $N$ admitting a Riemannian metric $g_{0}$ of non-positive curvature in case that $\pi_{1}(N)$ has a non-trivial center. It turns out that if $G$ attains the maximal dimension equal to the rank of Center $\pi_{1}(N)$ and the metric $g_{0}$ is real analytic, then any element of $G$ defining a diffemorphism homotopic to the identity of $N$ must be contained in the identity component $G^{0}$ of $G$. Moreover, if the inner automorphism group of $\pi_{1}(N)$ is torsion free, then the associated abstract kernel $\psi: G / G^{0} \rightarrow$ Out $\pi_{1}(N)$ is a monomorphism. The same result holds for the nonorientable $N$ 's under certain techical assumptions. Our result is an application of a theorem by Schoen-Yau (Topology, 18 (1979), 361-380) on harmonic mappings.
\end{abstract}

Mathematics Subject Classifications (2000): Primary: 57S25, 53C43; Secondary: 20F34.

Key words: aspherical manifold, compact Lie group action, non-positive curvature, harmonic mapping.

\section{Introduction}

Let $M$ be a closed aspherical manifold. A lot of information has been obtained by ConnerRaymond [2]-[4] regarding group actions on such manifolds. One of their main results in [2] is that a connected compact Lie group acting effectively and continuously on $M$ is a torus group of dimension not greater than the rank of Center $\pi_{1}(M)$ (note that $\pi_{1}(M)$ is torsion free by the Smith fixed point theorem). In particular, any compact Lie group acting effectively and continuously on a closed aspherical manifold with centerless fundamental group must be a finite group. A group action on $M$ is homotopically trivial if any element of this group, which defines a homeomorphism of $M$, is homotopic to the identity. Gottlieb-LeeÖzaydin [8] showed that a compact Lie group acting effectively and homotopically trivially on $M$ is abelian. A simple but nontrivial example of Gottlieb-Lee-Özaydin's result, is the group $\langle\exp (2 \pi \sqrt{-1} / m)\rangle \cong \mathbf{Z} / m \mathbf{Z}$ acting on the circle $S^{1}=\{\exp (\sqrt{-1} \theta): \theta \in[0,2 \pi)\}$ by multiplication. 
Let $G$ be a compact Lie group acting effectively and continuously on $M$ and $G^{0}$ be the identity component of $G$. We quickly review the definition of the associated abstract kernel that is a homomorphism $\psi: G \rightarrow$ Out $\pi_{1}(M)$ (cf Sections 2-3 [4] for the detail). Choose any base point $x$ in $M$ and for each $\alpha$ in $G$ select a path $\mathscr{P}_{\alpha}(t)$ joining $x$ to $\alpha x$. Define the corresponding automorphism $\alpha_{*}$ on $\pi_{1}(M, x)$ as follows: if $\sigma(t)$ is a loop based at $x$, then $\alpha_{*}(\sigma)$ is represented by

$$
\begin{cases}\mathscr{P}_{\alpha}(3 t) & 0 \leq t \leq 1 / 3 \\ \alpha(\sigma(3 t-1)) & 1 / 3 \leq t \leq 2 / 3 \\ \mathscr{P}_{\alpha}(3-3 t) & 2 / 3 \leq t \leq 1\end{cases}
$$

This $\alpha_{*}$ is unique up to an inner automorphism of $\pi_{1}(M, x)$ and yields a homomorphism $\psi: G \rightarrow$ Out $\pi_{1}(M)$, called the associated abstract kernel. Since $G^{0}$ is contained in the kernel of this homomorphism, $\psi$ can be reduced to another homomorphism from $G / G^{0}$ to Out $\pi_{1}(M)$, which we still call the associated abstract kernel and denote by $\psi$. A. Borel proved that, if $G$ is finite and $M$ has centerless fundamental group, then the associated abstract kernel $\psi: G \rightarrow$ Out $\pi_{1}(M)$ is a monomorphism (cf Theorem 3.2 [4]). In particular, the two homeomorphisms defined by any two elements of $G$ are not homotopic.

Lawson and Yau [10] showed that the isometry group $I(N)$ of a closed Riemannian manifold $N$ of non-positive curvature has dimension equal to the rank of Center $\pi_{1}(N)$, and that the identity component $I^{0}(N)$ of $I(N)$ is a torus group which is generated by the parallel vector fields on $N$ (in other words, every Killing vector field on $N$ is parallel). Obviously $I^{0}(N)$ acts homotopically trivially on $N$. But Lawson and Yau [10] proved that any homotopically trival element of $I(N)$ must be contained in $I^{0}(N)$.

Let $N$ be a closed smooth manifold admitting a Riemannian metric $g_{0}$ of non-positive curvature and $G$ be a compact Lie group acting smoothly and effectively on $N$. If $\pi_{1}(N)$ is centerless, then it follows from the results by Conner-Raymond and Borel that $G$ must be finite and the associated abstract kernel $\psi: G \rightarrow$ Out $\pi_{1}(N)$ is a monomorphism. In this paper, we shall investigate the following two problems:

Problem 1. When should any homotopically trivial element of $G$ be contained in $G^{0}$ (we are looking for a stronger version of Gottlieb-Lee-Özaydin's result for our case)?

Problem 2. To what extent Borel's theorem holds for the associated abstract kernel $\psi$ : $G / G^{0} \rightarrow$ Out $\pi_{1}(N)$ in case that $\pi_{1}(N)$ has a non-trivial center?

It turns out that these two problems are interconnected. By the result of Lawson-Yau, we are not missing any positive results if we assume that $G$ attains the maxiaml dimension equal to rank of Center $\pi_{1}(N)$. Since the fact that the inner automorphism group of $\pi(M)$ is torsion free is critical in the proof of Borel's theorem (cf [4]), it is naturally for us to assume this property for $\pi_{1}(N)$ while considering Problem 2.

Theorem 1.1. Let $N$ be a closed smooth manifold admitting a real analytic Riemannian metric $g_{0}$ of non-positive curvature and $G$ be a compact Lie group acting smoothly and effectively on $N$ and having positive dimension equal to the rank of Center $\pi_{1}(N)$. Suppose that there exists no free smooth action of odd prime order on $N$ if $N$ is non-orientable. Then any homotopically trivial element of $G$ must be contained in $G^{0}$. Moreover, if the inner automorphism group of $\pi_{1}(N)$ is torsion free, then the associated abstract kernel $\psi: G \rightarrow$ Out $\pi_{1}(N)$ is a monomorphism. 
Remark 1.1. The previous theorem only gives an incomplete answer to those two problems for non-orientable $N$ 's. For an example, the only non-orientable closed 2-manifold whose fundamental group has non-trivial center is the Klein bottle $K$. Since there exists no nontrivial free action on $K$, we know the answer of Problem 1 for the Klein bottle $K$ is Yes. However, the author does not know the answer for $K \times S^{1}$. Since there exists an involutive inner automorphism of $\pi_{1}(K)$, the theorem can not give the answer of Problem 2 for the Klein bottle $K$, either. But the author conjectures that the assumption for non-orientable $N$ 's is removable.

If a compact Riemannian manifold has non-positive curvature and has a point at which all sectional curvatures are negative, then Sampson in Theorem 5 of [11] used his rigidity theorem (cf Theorem 4, [11]) of harmonic mappings to show that the isometry group of the Riemannian manifold is finite, and no two of its elements are homotopic. Here we call a harmonic map is rigid if it is the only harmonic map in its homotopy class. Due to Frankel [6], the same result as Sampson's also holds for a compact Riemannian manifold of nonpositive curvature and of negative definite Ricci tensor. We shall not only unify the results of Sampson and Frankel from the point of view of Borel's theorem, but also give a stronger result than theirs in the following

Theorem 1.2. Let $\left(V, g_{1}\right)$ be a closed Riemannian manifold of non-positive curvature and having a point at which the Ricci tensor is negative definite. Then the following two statements hold:

a. $\pi_{1}(V)$ is centerless.

b. Any compact Lie group $G$ acting effectively and smoothly on $V$ is finite and the associated abstract kernel $\psi: G \rightarrow$ Out $\pi_{1}(N)$ is a monomorphism.

A QUICK PROOF OF THEOREM 1.2. It follows from a theorem of Bochner [1] (also see pages 324-326 in [13]) that every Killing field on $V$ is zero since $V$ has non-positive Ricci curvature and has a point at which the Ricci tensor is negative definite. Hence the isometry group of $\left(V, g_{1}\right)$ is finite. By the result of Lawson and Yau, $\pi_{1}(N)$ is centerless. Then by the result of Conner-Raymond, the compact Lie group $G$ must be a finite group even if $G$ only acts continuously on $N$. By Borel's theorem the associated abstract kernel $\psi: G \rightarrow$ Out $\pi_{1}(N)$ is a monomorphism.

This paper is organized as follows. In Section 2 we cite the rigidity theorem of SchoenYau [12] and prove a rigidity theorem (cf Theorem 2.1]) of the harmonic mappings to compact Ricci quasi-negatively curved manifolds. The first of them will be used to prove Theorem 1.1. and the second will be applied to an analytic proof of Theorem 1.2. In Section 3, we prepare two lemmata for the proof of these two theorems. The last section of this paper consists of the proof of Theorem 1.1 and the analytic proof of Theorem 1.2 .

\section{Rigidity theorems of harmonic mappings}

Theorem SY (cf Theorem 4 in Schoen-Yau [12]) Suppose $M, N$ are compact connected real analytic Riemannian manifolds and $N$ has nonpositive curvatures. Suppose $h: M \rightarrow N$ is a surjective harmonic map and its induced map $h_{*}: \pi_{1}(M) \rightarrow \pi_{1}(N)$ is also surjective. Then the space of surjective harmonic maps homotopic to $h$ is represented by $\{\beta \circ h \mid \beta \in$ $\left.I^{0}(N)\right\}$. 
Theorem 2.1. Let $h_{0}: M \rightarrow N$ be a harmonic mappings, where $M$ is compact and $N$ of nonpositive sectional curvature. Suppose that there exists a point $p$ in $M$ such that the followings hold:

(a) at $h_{0}(p)$ the Ricci tensor of $N$ is negative definite,

(b) the differential map $d h_{0}(p)$ of $h_{0}$ at $p$ is surjective.

Then $h_{0}$ is the only harmonic mapping in its homotopy class.

Before the proof of the above theorem, we make a quick review on the formula for second variation of the energy (cf J. Jost [9]) for a family of harmonic mappings.

Let $M$ be a compact, and $N$ a complete Riemannian manfiolds of dimension $m$ and $n$, respectively. In local coordinates, the metric tensor of $M$ is written as $\left(\gamma_{\alpha \beta}\right)_{\alpha, \beta=1, \cdots, m}$, and the one of $N$ as $\left(g_{i j}\right)_{i, j=1, \cdots, n}$. We shall use the notation $\left(\gamma^{\alpha \beta}\right)_{\alpha, \beta=1, \cdots, m}=\left(\gamma_{\alpha \beta}\right)_{\alpha, \beta=1, \cdots, m}^{-1}$ (inverse metric tensor). Let $f: M \rightarrow N$ be a smooth map and $f^{-1} T N$ the pullback bundle on $M$ by $f$ of the tangent bundle $T N$ of $N . f^{-1} T N$ has the metric $\left(g_{i j}(f(x))\right.$, and the cotangent bundle $T^{*} M$ of course has the metric $\left(\gamma^{\alpha \beta}\right)$. Then the energy density $e(f)$ is defined as $\frac{1}{2}\|d f\|^{2}$, which is the square of the norm of the differential of $f$ as a section of the Riemannian bundle $T^{*} M \otimes f^{-1} T N$. The energy of $f: M \rightarrow N$ is $E(f):=\int_{M} e(f) d M$, with $d M$ the volume form of $M$. The smooth map $f: M \rightarrow N$ is harmonic if and only if it is a critical point of the energy functional $E$.

Let $F: M \times(-\varepsilon, \varepsilon) \rightarrow N, F_{t}(x)=F(x, t)$ be a family of smooth maps between Riemannian manfiolds, in which $F_{0}(x)=F(x, 0)=f$. Then $W:=\left.\frac{\partial F}{\partial t}\right|_{t=0}$ is a section of $f^{-1} T N$. Let $\nabla$ denote the Levi-Civita connection in $f^{-1} T N$ and $R^{N}$ the curvature tensor of $N$. Then we have the following

Fact $\mathbf{E}$ For the second variation of energy the equality

$$
\left.\frac{\partial^{2}}{\partial t^{2}} E\left(F_{t}\right)\right|_{t=0}=\int_{M}\|\nabla W\|_{f^{-1} T N}^{2} d M-\int_{M} \operatorname{trace}_{M}\left\langle R^{N}(d f, W) W, d f\right\rangle_{f^{-1} T N} d M
$$

holds provided that $F(x, \cdot)$ is geodesic for every $x$.

Then we recall a result on homotopic harmonic mappings by Hartman [7].

Fact $\mathbf{H}$ (cf [9]) Assume that $N$ is a complete manifold of nonpositive sectional curvature. Let $f_{0}, f_{1}: M \rightarrow N$ be homotopic harmonic mappings. Then there exists a family $f_{t}: M \rightarrow N, t \in[0,1]$, of harmonic mappings connecting them, for which the energy $E\left(f_{t}\right)$ is independent of $t$, and for which every curve $\gamma_{x}(t):=f_{t}(x)$ is geodesic, and $\left\|\frac{\partial}{\partial t} \gamma_{x}(t)\right\|$ is independent of $x$ and $t$.

ProOF OF THEOREM 2.1 Let $h_{1}: M \rightarrow N$ be a harmonic map homotopic to $h_{0}$. We can find a family $h_{t}: M \rightarrow N, t \in[0,1]$, of harmonic mappings connecting them with the property as Fact $\mathrm{H}$. By Fact E, since $N$ has nonpositive curvature,

$$
\begin{aligned}
0 & =\frac{d^{2}}{d t^{2}} E\left(h_{t}\right) \\
& =\int_{M}\left(\left\|\nabla \frac{\partial}{\partial t} \gamma_{x}(t)\right\|^{2}-\operatorname{trace}_{M}\left\langle R^{N}\left(d h_{t}(x), \frac{\partial}{\partial t} \gamma_{x}(t)\right) \frac{\partial}{\partial t} \gamma_{x}(t), d h_{t}(x)\right\rangle_{h_{t}^{-1} T N}\right) d M \\
& \geq 0 .
\end{aligned}
$$


Hence we obtain that for any $x \in M$ and any $t \in[0,1]$,

$$
\operatorname{trace}_{M}\left\langle R^{N}\left(d h_{t}(x), \frac{\partial}{\partial t} \gamma_{x}(t)\right) \frac{\partial}{\partial t} \gamma_{x}(t), d h_{t}(x)\right\rangle_{h_{t}^{-1} T N}=0
$$

in particular,

$$
\operatorname{trace}_{M}\left\langle R^{N}\left(d h_{0}(p), \frac{\partial}{\partial t} \gamma_{p}(t)\right) \frac{\partial}{\partial t} \gamma_{p}(t), d h_{0}(p)\right\rangle_{h_{0}^{-1} T N}=0
$$

By assumptions (a), (b) and the following Fact T, we can see that the tangent vector $\frac{\partial}{\partial t} \gamma_{p}(t)$ of the geodesic $\gamma_{p}(t)$ vanishes. By Fact $\mathrm{H}$ every geodesic $\gamma_{x}(t), x \in M, t \in[0,1]$ degenerates into a point. That is, $h_{0}=h_{1}$.

Fact $\mathbf{T}$ Let $R$ be a $n \times n$ real symmetric matrix having nonpostive eigenvalues and negative trace. Suppose $B$ is a $m \times n$ real matrix with rank $n$. Then the matrix $B R B^{t}$ also has negative trace, where $B^{t}$ is the transposed matrix of $B$.

ProOF We may assume $R$ to be the diagonal matrix $\operatorname{diag}\left(l_{1}, \cdots, l_{n}\right)$, in which $l_{1}<0$ and $l_{2}, \cdots, l_{n} \leq 0$, without loss of generality. We denote $B=\left(b_{i j}\right)_{1 \leq i \leq m, 1 \leq j \leq n}$. Since $B$ has rank $n$, there exists a nonzero element $b_{i 1}$ in the first row of $B$. By computation, we know trace $B R B^{t} \leq l_{1} b_{i 1}^{2}<0$.

\section{Two lemmata}

Lemma 3.1. Let $M$ and $N$ be compact connected smooth Riemannian manifolds of the same dimension and $f: M \rightarrow N$ a smooth mapping. Assume deg $f=m \neq 0$ if $M$ is orientable, and $\operatorname{deg} f \equiv 1(\bmod 2)$ if $M$ is non-orientable. Define a subgroup A of $I(M)$ by

$$
A=\{\alpha \in I(M) \mid f \circ \alpha=f\} .
$$

Then if $M$ is orientable, the order of $A$ divides $m$. In particular, in case of deg $f= \pm 1$, the group $A$ is trivial. If $M$ is non-orientable, the order of $A$ is an odd integer.

Proof. We firstly prove the part in which $M$ is orientable. Taking a regualr value $y_{0} \in N$ whose preimages under $f$ are $x_{1}, \cdots, x_{2 k+m}$, then

$$
m=\operatorname{deg} f=\Sigma_{i=1}^{2 k+m} \operatorname{sgn} \operatorname{det} J f\left(x_{i}\right),
$$

where $J f\left(x_{i}\right)$ is the Jacobian matrix of $f$ at point $x_{i}$. Without loss of generality, we set $m \geq 1$ and

$$
\operatorname{sgn} \operatorname{det} J f\left(x_{i}\right)=1 \text {, for } 1 \leq i \leq k+m \text {; }
$$

$$
\text { sgn det } J f\left(x_{j}\right)=-1 \text {, for } k+m+1 \leq j \leq 2 k+m \text {. }
$$

The group $A$ acts on the set $f^{-1}\left(y_{0}\right)$ by the definition of $A$. We claim that $A$ acts freely on this set. In fact if an element $\beta \in A$ has $x_{1}$ as its fixed point, then its differential at point $x_{1}$ is the identity map since $d f\left(x_{1}\right) \circ d \beta\left(x_{1}\right)=d f\left(x_{1}\right)$. Since $M$ is connected, $\beta$ must be the identity of $M$.

We also claim that $A$ should preserve the orientation of $M$. Otherwise, letting $\bar{A}$ be the subgroup of $A$ whose elements preserve the orientation of $M$, we have $[A: \bar{A}]=2$. If we set $\bar{A}=\left\{g_{1}, \cdots, g_{n}\right\}, A=\left\{g_{1}, \cdots, g_{n}, g g_{1}, \cdots, g g_{n}\right\}$, then the equalities

$$
\text { sgn det } J f(x)=\operatorname{sgn} \operatorname{det} J f\left(g_{i}(x)\right), \operatorname{sgn} \operatorname{det} J f(x)=-\operatorname{sgn} \operatorname{det} J f\left(g g_{i}(x)\right)
$$


hold for any $x \in f^{-1}\left(y_{0}\right)$ and $1 \leq i \leq n$. As $A$ acts freely on $f^{-1}\left(y_{0}\right)$, we obtain $\operatorname{deg} f=0$ and a contradiction. Since $A$ preserves the orientation of $M$ and acts freely on the set $\left\{x_{1}, \cdots, x_{2 k+m}\right\}, A$ should act freely on the two set $\left\{x_{1}, \cdots, x_{k+m}\right\}$ and $\left\{x_{k+m+1}, \cdots, x_{2 k+m}\right\}$ respectively so that the order of $A$ divides $m$.

When $M$ is non-orientable, we know the number of the set $f^{-1}\left(y_{0}\right)$ is odd because of $\operatorname{deg} f \equiv 1(\bmod 2)$. By the same way, we can show that $A$ acts freely on $f^{-1}\left(y_{0}\right)$ so that the order of $A$ is also odd.

Remark 3.1. Schoen and Yau proved a result (cf. Theroem 4 (i) of [12]) more general than Lemma 3.1. However we prefer a simple proof as above for our special case.

We also need a well known result as follows:

Lemma 3.2. Let $M$ be a non-orientable smooth manifold and $M^{\prime}$ its orientable double covering. Then a diffeomorphism of $M$ can be lifted to that of $M^{\prime}$.

\section{Proof of Theorems 1.1 and 1.2}

Proof of TheOREM 1.1 Let $r>0$ be the rank of Center $\pi_{1}(N)$. Then $I^{0}\left(N, g_{0}\right)$ is a torus group of dimension $r$. Since $G$ is a compact Lie group acting smoothly on $N$, we can choose a real analytic Riemannian metric $g$ such that $G$ acts on $(N, g)$ isometrically. By Eells-Sampson [5] We have a harmonic mapping $h:(N, g) \rightarrow\left(N, g_{0}\right)$ homotopic to the identity. Let $H$ be the subgroup of $G$ consisting of elements homotopic to the identity map of $N$. Since $H$ contains $G^{0}, H$ is a closed Lie subgroup of $G$ having the same dimension as $G$. For an element $\alpha$ of $H$, since $h$ and $h \circ \alpha$ are two homotopic harmonic mappings satisfying the condition of Theorem SY, there exists a unique element $\beta$ of $I^{0}\left(N, g_{1}\right)$ such that $h \circ \alpha=\beta \circ h$, which leads to a Lie group homomorphism

$$
\rho: H \rightarrow I^{0}\left(N, g_{1}\right), \rho(\alpha):=\beta \text {. }
$$

We shall prove $H=G^{0}$ in the following.

Case 1 If $N$ is orientable, then we have a monomorphism $\rho: H \rightarrow I^{0}\left(N, g_{0}\right)$ by Lemma 3.1. Remember that $I^{0}\left(N, g_{0}\right)$ is a torus group of dimension equal to rank of Center $\pi_{1}(N)$. Sine $\operatorname{dim} G=\operatorname{dim} I^{0}\left(N, g_{1}\right), \rho$ must be an isomorhism so that $H$ is a torus group. But $H$ contains the tours group $G^{0}$ with the same dimension, so $H=G^{0}$.

Case 2 Let $N$ be non-orientable. Suppose that Kernel $\rho$ is non-trivial. By Lemma 3.1 there exists an element $\alpha$ of Ker $\rho$ whose order is an odd prime number. If $\alpha$ has a fixed point $x$ on $N$, by Corollary 6.2 in [2] $\alpha_{*}$ induces a non-trivial automorphism of $\pi_{1}(N, x)$, which contradicts that $\alpha$ is homotopic to the identity map of $N$. Therefore, $\alpha$ acts freely on $N$, which contradict the assumption that there exists no free smooth action of odd prime order on $N$.

By now we have showed that any homotopically trivial element of $G$ is contained in $G^{0}$. Finally we prove the associate abstract kernel $\psi: G / G^{0} \rightarrow$ Out $\pi_{1}(N)$ is a monomorphism if the inner automorphism group Inn $\pi_{1}(N)$ is torsion free. By previous argument, we need only to show that an element $[\alpha]$ in $G / G^{0}$ inducing an inner automorphism of $\pi_{1}(N)$ is homotopically trivial. Indeed, since $[\alpha]$ is of finite order and the inner automophism group 
of $G$ is torsion free, $\psi([\alpha])$ must be the identity map on $\pi_{1}(N)$. Since $N$ is a $K\left(\pi_{1}(N), 1\right)$ space, $\alpha$ is homotopic to the identity map of $N$.

Proof of THEOREM 1.2 At first we prove the finiteness of $G$. We only need to show that the identity component $G^{0}$ is trivial. Since $G$ is a compact Lie group acting on $N$, we can choose a Riemannian metric $g$ such that $G$ action on $(V, g)$ is isometric. By Eells-Sampson [5], there exists a harmonic mapping $h:(V, g) \rightarrow\left(V, g_{1}\right)$ homotopic to the identity so that $h$ is surjective. We shall prove

Claim $1 h$ is the only harmonic mapping in its homotopy class.

Proof of Claim 1 Since $\left(V, g_{1}\right)$ has a point at which the Ricci tensor is negative definite, there exists an open subset $U$ of $N$ on which $\left(V, g_{1}\right)$ has negative definite Ricci tensor. By Sard's theorem and the surjectivity of $h$, we know the regular value set of $h$ is a dense subset of $N$ so that there exists a regular point of $h$ which is mapped into $U$. Then we apply Theorem 2.1.

By Claim $1 G^{0}$ is contained in the set $\{\alpha \in G: h \circ \alpha=h\}$, which is a finite set by Lemma 3.1. That is, $G^{0}$ is trivial.

Then we prove that no two elements of $G$ are homotopic. We only need to prove that an element $\alpha$ of $G$ homotopic to the identity is the identity. If $V$ is orientable, then by Claim 1 and Lemma 3.1 it follows from the fact that $h$ has degree 1. In the following we may assume that $V$ is non-orientable. Let $V^{\prime}$ be its orientable double covering space and $\gamma: V^{\prime} \rightarrow V^{\prime}$ the deck transformation. By Lemma 3.2 $\alpha$ has two liftings $\tilde{\alpha}, \gamma \circ \tilde{\alpha}$ on $V^{\prime}$, in which $\tilde{\alpha}$ is homotopic to $\operatorname{id}_{V^{\prime}}$. Since the order of $\tilde{\alpha}$ is equal to that of $\alpha \leq|G|$, applying the previous argument to the finite group action on $V^{\prime}$ generated by $\tilde{\alpha}$, we can see that $\tilde{\alpha}$ is id $V_{V^{\prime}}$ and then $\alpha$ also should be the identity of $V$.

Claim $2 \pi_{1}(V)$ is centerless.

PROOF OF CLAIM 2 It has been proved in Introduction. We give an alternative proof as follows. The previous argument tells us that $I\left(V, g_{1}\right)$ is a finite group. By the result of Lawson and Yau mentioned in Introduction, the center of $\pi_{1}(V)$ has rank zero. The proof is completed by the well known fact that $\pi_{1}(V)$ is torsion free.

Finally we show that the abstract kernel $\psi: G \rightarrow$ Out $\pi_{1}(V)$ is a monomorphism. We have to prove that an element $\alpha$ of $G$ inducing an inner automorphism $\imath(\alpha)$ of $\pi_{1}(V)$ must be the identity map of $V$. By Claim 2, the inner automorphism group Inn $\pi_{1}(V)$ of $\pi_{1}(V)$ is isomorphic to $\pi_{1}(V) /$ Center $\pi_{1}(V)=\pi_{1}(V)$, which is torsion free. Since $\alpha$ is of finite order and Inn $\pi_{1}(V)$ is torsion free, the inner automorphism $\imath(\alpha)$ induced by $\alpha$ must be the identity map of $\pi_{1}(V)$. Since $V$ is a $K\left(\pi_{1}(V), 1\right)$-space, the diffeomorphism $\alpha$ on $V$ must be homotopic to the identity map of $V$. The previous argument gives us that $\alpha$ should equal the identity map of $N$.

\section{Acknowledgements}

This study was supported in part by the Japanese Government Scholarship, the JSPS Postdoctoral Fellowship for Foreign Researchers and the FRG Postdoctoral Fellowship. 


\section{References}

[1] S. Bochner, Vector Fields and Ricci Curvature, Bull. Amer. Math. Soc. 52 (1946), 776-797.

[2] P. Conner And F. Raymond, Actions of Compact Lie Groups on Asperical Manifolds, in Topology of Manifold (Proceedings of the University of Georgia Topology of Manifolds Institute), ed. J. C. Cantrell and C. H. Edwards, Jr. Markham, (1970), 227-264

[3] P. Conner And F. RAYMOnd, Injective Operations of the Toral Groups, Topology, 10 (1971), 283-296

[4] P. Conner And F. Raymond, Manifolds with Few Periodic Homeomorphisms, in Lecture Notes in Math. 299, ed. H. T. Ku, L. N. Mann, J. L. Sicks and J. C. Su, Springer-Velag, New York, (1972), 1-75

[5] James Eells JR And J. H. SAmpson, Harmonic Mappings of Riemannian Manifolds, Amer. J. Math. 86 (1964), 109-160

[6] T. T. Frankel, On Theorem of Hurwitz and Bochner, J. Math. Mech. 15 (1966), 373-377

[7] P. Hartman, On Homotopic Harmonic maps, Can. J. Math. 19 (1967), 673-687

[8] D. H. Gottlieb And K. B. Lee And M. Özaydin, Compact Group Actions and Maps into $K(\pi, 1)$-Space, Trans. Amer. Math. Soc 287 (1985), 419-429

[9] J. Jost, Riemannian Geometry and Geometric Analysis, 3rd edition, Springer, 2000.

[10] H. B. LAwson And S. T. YAU, Compact Manifolds with Nonpositive Curvature, J. Diff. Geo, 7 (1972), 211-228

[11] J. H. SAmpson, Some Properties and Applications of Harmonic Mappings, Ann. École Norm. Sup. (4) 11 (1978), no. 2, 211-228

[12] R. Schoen And S. T. YAU, Compact Group Actions and the Topology of Manifolds with Nonpositive Curvature, Topology, 18 (1979), 361-380

[13] Hung-Hsi Wu, The Bochner Technique in Differential Geometry, Math. Rep. 3 (1988), no. 2, 289-3538. 